\title{
Seizure Prophylaxis in Neurosurgery Settings: Role of Prophylactic Brivaracetam in Neurosurgery
}

\author{
Paresh K Doshi ${ }^{1}$, Manas Panigrahi ${ }^{2}$, Arundhuti Ghosh ${ }^{3}$, Kumar Gaurav ${ }^{4 *}$ and Sukhpreet Singh ${ }^{5}$ \\ ${ }^{1}$ Director of Neurosurgery and Functional Neurosurgery, Jaslok Hospital and Research Centre, India \\ ${ }^{2}$ Senior Consultant Neurosurgeon, KIMS, India
}

${ }^{3}$ Medical Advisor - Medical Affairs, Dr. Reddy's Laboratories Ltd, India

${ }^{4}$ Medical Affairs, Dr. Reddy's Laboratories Ltd, India

${ }^{5}$ Dr. Reddy's Laboratories Ltd, India

*Corresponding author: Kumar Gaurav, Director- Medical Affairs, Dr. Reddy's Laboratories Ltd, Hyderabad, India

\section{ARTICLE INFO}

Received: 幽 August 24, 2021

Published: 轓 August 30, 2021

Citation: Paresh KDoshi, Manas Panigrahi, Arundhati Ghosh, Kumar Gaurav, Sukhpreet Singh. Seizure Prophylaxis in Neurosurgery Settings: Role of Brivaracetam Prophylactic Brivaracetam in Neurosurgery. Biomed J Sci \& Tech Res 38(3)-2021. BJSTR. MS.ID.006154.

Keywords: Prophylaxis; Brivaracetam; Neurosurgery

\begin{abstract}
Background: Seizures are common sequelae to cranial surgical interventions for tumors, subarachnoid hemorrhage, traumatic brain injury, cerebral venous thrombosis, and stroke. In the post-operative period, seizures are a marker of poor outcomes and impaired quality of life.

Methods: A panel of ten neurosurgery experts from India shared clinical experiences for seizure prophylaxis in neurosurgical settings, reviewed efficacy and safety of brivaracetam, and discussed the drivers and barriers for adopting brivaracetam in the neurosurgical settings.

Results: About $60-70 \%$ of patients in the neurosurgical intensive care need prophylaxis for seizures and levetiracetam is the preferred choice. Other antiepileptic agents used for prophylaxis include phenytoin and lacosamide. Adverse events, including sedation, behavioral changes, and cognitive decline are commonly associated with antiepileptic prophylaxis and guide the selection of antiepileptic drugs. Brivaracetam, the first selective ligand for synaptic vesicle protein $2 \mathrm{~A}$, has a better safety profile than other antiepileptics including levetiracetam. With high lipophilicity and favorable pharmacokinetics, brivaracetam can be initiated at target doses without titration. Oral brivaracetam has been extensively used an add-on agent for a long-term chemoprophylaxis of seizures.
\end{abstract}

Conclusion: Brivaracetam has clinical benefits for seizure prophylaxis in neurosurgical settings. However, prohibitive cost and lack of an intravenous formulation may limit the clinical utility of brivaracetam in a developing country like India.

\section{Introduction}

Seizures are a common challenge in neurosurgery practice. The risk of post-craniotomy seizures varies from $3 \%$ to $92 \%$ over five years [1]. About $20-50 \%$ patients have at least one seizure in the post-operative period and about two-thirds of seizures occur within the first month of cranial surgery [2,3]. There are differences in the duration of prophylaxis and choice of an antiepileptic drug because of insufficient evidence [4-6]. Newer antiepileptic could 
change the landscape of seizure prophylaxis in neurosurgical settings. Brivaracetam, approved in 2016 by the FDA for adjunctive treatment of focal seizures $[7,8]$, is approved in India an adjunctive therapy for partial-onset seizures.

\section{Methodology}

A 'medical consensus' was developed to use brivaracetam in neurosurgery with invited experts who reviewed available evidence for brivaracetam and shared their clinical experience to synthesize state-of-the-art knowledge. Agreement of $80 \%$ of the panel was considered as unanimous for the development of consensus and formulation of a recommendation. Recommendations of the expert panels and quality of evidence (High, medium, low) were graded in accordance with the US Preventive Services Task Force Ratings for the magnitude of net benefit with brivaracetam in clinical experience. The quality of evidence was graded as high, medium, and low [9]. An expert panel convened a discussion to reflect on the experience and outcomes of seizure prophylaxis in patients who undergo neurosurgical procedures. The panel included experts from key neurosurgery centers in India who were chosen for their vast experience and interest in seizure prophylaxis. The meeting was held in, India on May, 2020. Experts shared their clinical experiences for seizure prophylaxis in neurosurgical settings and reviewed the efficacy and safety of brivaracetam. The panel also discussed the drivers and barriers for the adoption of brivaracetam in the neurosurgical settings.

\section{Seizures in Neurosurgery Patients}

In neurosurgical practice, seizures are a common challenge in patients with tumors, subarachnoid hemorrhage, traumatic brain injury, cerebral venous thrombosis, and stroke. Seizures are a marker of poor outcomes in patients with subarachnoid hemorrhage [10]. Acute symptomatic seizures are reported in 6.9-76\% patents with cerebral venous and sinus thrombosis [11]. Post-stroke seizures are a marker for poor outcome [12]. Seizures are common in patients with brain tumors including glioneuronal tumors, gliomas, meningiomas, and brain metastases [13].

Pharmacoprophylaxis for Seizures: The American Heart Association (AHA) recommends using prophylactic anticonvulsants in the immediate post hemorrhagic period after subarachnoid hemorrhage. Routine long-term use of anticonvulsants is not routinely recommended though it may be adopted in patients with risk factors for delayed seizure disorders. These include patients with past history of seizure, intracerebral hematoma, intractable hypertension, infarction, or aneurysm at the middle cerebral artery [14]. In patients with traumatic brain injury, antiepileptic drugs are indicated within seven days of the injury [15]. Primary prophylaxis in acute symptomatic seizures is not recommended in patients with cerebral venous thrombosis. However, prophylaxis may be initiated after the first seizure in patients with supratentorial lesions or focal neurological deficits [11]. Post-stroke seizures are treated with anti-epileptic drugs if recurrent. However, prophylactic use of antiepileptic drugs in these patients is not routine [12]. Guidelines by the Brain Trauma Foundation recommend pharmacoprophylaxis against early seizures following brain trauma [16].

Expert Consensus: Of all neurosurgical procedures, 80-90\% are supra tentorial in location and seizure prophylaxis should be considered for such procedures.

\section{Brain Tumors and Seizures}

Seizures are common in patients with brain tumors and may be the presenting symptom in various tumors. Seizures are most common in patients with glioneuronal tumors (70-80\%), low grade gliomas (60-75\%), meningiomas (20-50\%), and brain metastasis (20-35\%). Surgical intervention abolishes seizures in about 60$90 \%$ patients [13]. Anti-epileptic drugs are key to management of seizures in patients undergoing surgery for brain tumors. The choice of AED is influenced by various factors including patient characteristics (age, comorbidities, and organ dysfunction) and concurrent chemotherapy. Enzyme-inducing antiepileptic drugs should be avoided to prevent any drug interactions with ongoing chemotherapy [17]. Monotherapy or combined therapy with levetiracetam and valproate is commonly used for the treatment of seizures in patients with brain tumors. In a retrospective analysis, $77.7 \%, 69.5 \%$, and $60.3 \%$ patients on levetiracetam, valproic acid, and combination of the two, respectively were seizure free after a median follow up of nine months [18]. Evidence from published studies suggests that some surgeons select lacosamide as add-on agent when there is inadequate response to levetiracetam and valproate either alone or in combination [19].

Prophylactic antiepileptic drugs help in preventing seizures in patients with brain tumors who do not have a history of seizures. Such patients are candidates for short-term prophylaxis after surgery for cerebral tumors [20]. In a prospective, randomized phase II study, patients with supratentorial tumors received levetiracetam $(n=73)$ or phenytoin $(n=73)$ as prophylaxis for seven days after craniotomy. The incidence of seizures was significantly less in the patients who received levetiracetam vs. phenytoin $(1.4 \%$ vs. $15.1 \%, p=0.005$ ) and the odds ratio for being seizure free with levetiracetam vs, phenytoin was 12.77 (95\% CI 2.39 to 236.71, $\mathrm{p}=0.001$ ). In this study, all patients completed levetiracetam prophylaxis whereas five patients who received phenytoin reported discontinuation of treatment due to adverse events [21]. However, in a meta-analysis (1983-2015) of ten trials $(n=1815)$, there was limited evidence to support the prophylactic use of antiepileptic drugs in post craniotomy seizures [22].

Antiepileptic prophylaxis is commonly used in patients with 
brain tumors [23,24]. and most clinicians use levetiracetam in practice, some clinicians continue a longer-term prophylaxis (>one year) for patients who undergo surgery for cerebral tumors and have history of seizures. Brivaracetam prophylaxis should be considered in patients who develop cognitive impairment after neurosurgical procedures. It is usually preferred in patients who develop behavioral disturbances with levetiracetam. Brivaracetam, devoid of any hepatic enzyme inducing effects, is also safe for use in patients with brain tumors who undergo chemotherapy. Brivaracetam and lacosamide have shown in-vitro dose-dependent cytotoxic and anti-migratory effects in human glioma cell lines [25]. Suggestive of potential anti-neoplastic effects, these properties may offer additional clinical benefits in patients with brain tumors.

Expert Consensus: Patients with malignancy who have a history of seizures may be given a longer-term prophylaxis for more than a year. Any patient who has history of seizure or who developed seizure in peri-operative period, prophylaxis may be continued for two years post seizure. Levetiracetam is the most commonly used antiepileptic prophylaxis in patients who undergo surgery for brain tumors. Brivaracetam is a good prophylactic option for longer term prophylaxis and also for patients who show cognitive impairment after craniotomy for cerebral tumors.

\section{Anti-Epileptic Drugs for Prophylaxis}

About $60-70 \%$ of patients in the neurosurgical intensive care need prophylaxis for seizures and levetiracetam is the drug of choice. Antiepileptic prophylaxis is initiated at loading dose before conclusion of the surgical procedure. The preferred agents are levetiracetam or phenytoin. In patients with tumors who develop seizures while on levetiracetam, lacosamide is the preferred agent. During the immediate post-operative period the patient is monitored for central functions and can be transitioned from phenytoin/levetiracetam to oral brivaracetam. There are evident trends for the shift from phenytoin to levetiracetam for seizure prophylaxis in the neurosurgery intensive care [26]. In a metaanalysis of three studies $(n=243)$, levetiracetam prophylaxis resulted in fewer seizures when compared to phenytoin $(\mathrm{OR}=0.12$ [0.03-0.42]: $\chi 2=1.76: \mathrm{I} 2=0 \%$ ) in adult patients with brain tumor who underwent supratentorial craniotomy for tumor resection or biopsy [27]. In a prospective randomized study, patients with severe traumatic brain injury $(n=46)$ or subarachnoid hemorrhage $(\mathrm{n}=\mathrm{six})$ who received levetiracetam $(\mathrm{n}=34)$ had similar incidence of early seizures, seizures at six months, or mortality as those who received phenytoin $(n=18)$.

However, there was a significantly better Disability Rating
Score at three and six months in patients receiving levetiracetam [28]. In another study, 32 patients with severe traumatic brain injury received levetiracetam for the first seven days and were followed prospectively for abnormal EEG findings (classified as status epilepticus, seizure activity, or seizure tendency). Data were compared with a historical cohort of 41 patients with severe traumatic brain injury who received phenytoin. No significant differences in seizure activity were found on EEG between patients receiving levetiracetam or phenytoin. However, levetiracetam monotherapy was associated with an increase in seizure tendency and increased epileptiform activity on EEG [29]. In a meta-analysis of 16 studies, levetiracetam had better efficacy than phenytoin $(\mathrm{OR}=0.63 ; 95 \% \mathrm{CI}=0.40-0.99 ; \mathrm{P}=.04)$ for prevention of early seizures after brain injury [30]. In some patients, two or more antiepileptic drugs may be needed. Due to the risk of seizure outbreak, some clinicians use levetiracetam as add-on therapy with other anti-epileptic drugs. A clinical advantage of levetiracetam over phenytoin is that it obviates the need for monitoring of serum levels. Levetiracetam has a better safety profile with lower frequency of worsened neurological status and gastrointestinal problems when compared to phenytoin [28]. Significantly fewer adverse events are reported for levetiracetam prophylaxis in patients undergoing surgery for brain tumor when compared to phenytoin or valproate [27].

Dosing for Pharmacoprophylaxis: Levetiracetam prophylaxis can be introduced with $500 \mathrm{mg}$ IV every 12 hours and the dose may be adjusted for therapeutic effects up to $1500 \mathrm{mg}$ IV every 12 hours (Maximum $3000 \mathrm{mg} /$ day). In patients with traumatic brain injury, a short-term prophylaxis may be given for up to six months. In some patients, prophylaxis may be discontinued after six weeks. Brivaracetam is administered as $50-200 \mathrm{mg}$ /day in two divided doses for prophylaxis.

Expert Consensus: Prophylaxis for seizures should be introduced early on when still planning a neurological surgery. Surgeries where pre-operative prophylaxis is not recommended or contraindicated, e.g. ventricular surgery where risk for seizures is less, prophylaxis can be initiated intra-operatively and continued post-operatively.

\section{Pharmacological Profile of Brivaracetam}

Brivaracetam has a distinct mechanism of action when compared to other antiepileptic drugs. It has 15- to 30-fold higher affinity for SV2A than levetiracetam [8]. Brivaracetam has no direct effect on $\alpha$-amino-3-hydroxy-5-methyl-4-isoxazolepropionic acid (AMPA), $\gamma$-aminobutyric acid (GABA), glycine, or kainic acid-gated 
currents. There is however a minor inhibitory action on $\mathrm{N}$-methyl d-aspartate (NMDA)-receptor activity at supratherapeutic Concentrations [30,31]. At therapeutic concentrations, there is no effect on ionic channels including the voltage-gated potassium and calcium channels $[32,33]$. Brivaracetam is well absorbed after oral dosing and has a linear kinetics. It is rapidly absorbed after single or multiple oral doses and has a median time to maximum concentration (tmax) of approximately one to two hours. The elimination $t 1 / 2$ is seven to eight hours. It undergoes metabolic clearance and has no induction potential on CYP3A4 activity [34] Brivaracetam has no drug-drug interactions.

Brivaracetam is well tolerated and only mild to moderate adverse events have been reported. The most common adverse events include dizziness and euphoria which are known to resolve within the first day of treatment. No clinically relevant changes are reported in laboratory tests, vital signs, physical examinations or cardiac monitoring [34]. The safety of brivaracetam spares the need for titration and allows initiation at target doses [35]. This along with the rapid entry into the brain in humans makes it a good candidate for seizure prophylaxis [36]. Brivaracetam has an early and sustained efficacy. In three phase III studies, time to onset of sustained $\geq 50 \%$ responder status was assessed for patients $(\mathrm{n}=1160)$ with focal (partial-onset) seizures who received placebo or adjunctive brivaracetam $50 \mathrm{mg} /$ day, $100 \mathrm{mg} /$ day, or $200 \mathrm{mg} /$ day [37]. Sustained $\geq 50 \%$ responder status on day one was seen in $6.7 \%$ patients receiving placebo, and $15.5 \%, 18.1 \%$, and $19.4 \%$ patients receiving the three doses of brivaracetam, respectively (all $\mathrm{P}<0.001$ vs placebo).

Efficacy of adjunctive brivaracetam spans across various subtypes of focal seizures including simple and complex partial seizures and secondarily generalized seizures in patients with uncontrolled seizures with one to two anti-epileptic drugs. In a phase III study in 760 patients, $\geq 50 \%$ responder rate (odds ratio vs. placebo; $95 \% \mathrm{CI}$ ) was $21.6 \%$ for placebo and $38.9 \%$ (2.39; 1.6-3.6; $\mathrm{p}<0.001)$ and $37.8 \%(2.19 ; 1.5-3.3 ; \mathrm{p}<0.001)$ for brivaracetam 100 $\mathrm{mg} /$ day and $200 \mathrm{mg} /$ day, respectively [37]. This broad spectrum of efficacy is a desirable feature for prevention of seizures after neurosurgical procedures. The effects of adjunctive BRV were evaluated on cognition and behavior in 43 patients with epilepsy in a naturalistic clinical setting with follow-up either after five days or after 25 weeks. There was a significant improvement in attention and executive functions ( $p=.03$ ) without an interaction with the length of the observation interval. At the longer-term follow-up, at least 50 percent reduction in seizure frequency was observed in $53 \%$ of the patients and $21 \%$ were seizure free [38].

Expert Consensus: Brivaracetam has a favorable pharmacokinetic profile and is well tolerated. Brivaracetam can be initiated at target doses without the need for titration. Brivaracetam has an early and sustained response which can be explained by the high lipophilicity leading to easy penetration of the blood brain barrier, high affinity for presynaptic SV2A proteins and greater occupancy of SV2A proteins, and a linear association between the SV2A affinity and anti-seizure properties.

\section{Brivaracetam vs. Levetiracetam}

Brivaracetam has good efficacy for pharmacoprophylaxis of seizures in neurosurgical settings. The differential interaction with SV2A may provide a molecular correlate to the distinct pharmacodynamic properties of brivaracetam and levetiracetam [9]. Brivaracetam has a faster onset of action when compared to levetiracetam. In a study in human volunteers $(n=9)$, drugentry half-time into the brain after a five minute IV infusion was two, ten, and 22 minutes with brivaracetam 200, 100, and $50 \mathrm{mg}$, respectively, compared with 20 minutes for levetiracetam $1500 \mathrm{mg}$ [36]. Brivaracetam is preferred in head injury patients as there are lesser chances of seizures in the post-operative period. Patients can be discharged with oral brivaracetam therapy. Brivaracetam is safer when compared to levetiracetam. Psychiatric adverse events are reported to occur in about $16 \%$ patients and are a common cause for treatment discontinuation for levetiracetam $[39,40]$. In patients who experience adverse events like behavioral problems, suicidal tendencies, excessive drowsiness, and psychosis with levetiracetam, switch to brivaracetam should be considered. In addition, brivaracetam may also be a better choice in patients who are overweight, or experience gain in weight with levetiracetam. At average drug load, brivaracetam has lesser cognition-related adverse effects and does not show a dose-dependent increase for these effects [41].

Adjunctive treatment with brivaracetam is associated with better effect on executive performance than levetiracetam. In a naturalistic clinical setting, brivaracetam was associated with a small $(>0.2)$ positive effect size (Cohen's $d=0.31$ ) on executive performance while a neutral effect (positive effect sizes $<0.2$ ) was seen for levetiracetam (Cohen's d = 0.14) [38]. Factors influencing the choice of levetiracetam over brivaracetam for seizure prophylaxis include the non-availability of intravenous formulation for brivaracetam and the high cost of brivaracetam.

Expert Consensus: Brivaracetam is used as an add-on drug for seizure prophylaxis in patients who undergo neurosurgical procedures. Brivaracetam is not used as first line drug as it is not available in intravenous formulation in India. Brivaracetam is safer than levetiracetam but cost is limiting. There is no dose-dependent increase in cognitive impairment with brivaracetam.

\section{Summary}

There was consensus for the use of brivaracetam for seizure prophylaxis in neurosurgical practices (Tables 1\&2). Postoperative seizures are a risk factor for development of epilepsy and adversely 
impact the quality of life in patients who undergo neurosurgical procedures. Seizure prophylaxis, when introduced early, helps to prevent seizures in patients in neurosurgical intensive care. Levetiracetam is the most commonly used pharmacoprophylaxis in neurosurgery. Other common drugs include phenytoin and lacosamide. Brivaracetam is a newer antiepileptic drug with a favorable pharmacological profile. Brivaracetam is the best antiepileptic drug for patients who experience cognitive impairment and behavioral problems with levetiracetam. Brivaracetam is preferred for prophylaxis rather than treatment of seizures who undergo neurological procedures.

Table 1: Expert consensus for the use of brivaracetam in neurosurgery practices.

\begin{tabular}{|c|c|c|c|c|c|}
\hline Statements & Agree & Neutral & Do not agree & Consensus & $\begin{array}{c}\text { Alternate } \\
\text { opinion, if any }\end{array}$ \\
\hline Brivaracetam has faster onset of action & $\sqrt{ }$ & & & & \\
\hline Brivaracetam does not require dose titration & & & & $\sqrt{ }$ & \\
\hline $\begin{array}{l}\text { Brivaracetam has broad spectrum of activity for } \\
\text { seizures following neurosurgical procedures }\end{array}$ & & & & $\sqrt{ }$ & \\
\hline $\begin{array}{l}\text { Brivaracetam has stronger affinity and potency than } \\
\text { levetiracetam towards SV2a glycoproteins }\end{array}$ & $\sqrt{ }$ & & & & \\
\hline Brivaracetam has high retention & $\sqrt{ }$ & & & & \\
\hline $\begin{array}{l}\text { Brivaracetam needs no dose adjustments in patients } \\
\text { with deranged renal functions }\end{array}$ & $\sqrt{ }$ & & & & \\
\hline Brivaracetam has a good tolerability profile & & & & $\sqrt{ }$ & \\
\hline $\begin{array}{l}\text { Brivaracetam is associated with lesser adverse } \\
\text { events when compared to other antiepileptics }\end{array}$ & & & & $\sqrt{ }$ & \\
\hline $\begin{array}{l}\text { Brivaracetam does not adversely impact cognitive } \\
\text { functions }\end{array}$ & & & & $\sqrt{ }$ & \\
\hline No drug interactions are reported with brivaracetam & & & & $\sqrt{ }$ & \\
\hline $\begin{array}{c}\text { Young college going female with POS and secondary } \\
\text { generalisation is a candidate for brivaracetam } \\
\text { therapy }\end{array}$ & & & & $\sqrt{ }$ & \\
\hline $\begin{array}{l}\text { Middle aged male with localised epilepsy and high } \\
\text { seizure frequency is a candidate for brivaracetam } \\
\text { therapy }\end{array}$ & & & & $\sqrt{ }$ & \\
\hline $\begin{array}{l}\text { An elderly patient with multiple comorbidities and } \\
\text { receiving concomitant medications is a candidate for } \\
\text { brivaracetam therapy }\end{array}$ & & & & $\sqrt{ }$ & \\
\hline
\end{tabular}

Table 2: List of the panel members.

\begin{tabular}{|c|c|c|}
\hline Name of expert & Specialty & City \\
\hline Dr Nitin Dange & Neurosurgeon & Mumbai \\
\hline Dr.Darpan Thakre & Neurosurgeon & Mumbai \\
\hline Dr.Paresh Doshi & Neurosurgeon & Mumbai \\
\hline Dr. Vivek Agarwal & Neurosurgeon & Mumbai \\
\hline Dr. Rajesh Jain & Neurosurgeon & Chhattisgarh \\
\hline Dr. Suryaprakash Gora & Neurosurgeon & Delhi \\
\hline Dr. K K Bansal & Neurosurgeon & Raj/UP \\
\hline Dr. Amit Ghosh & Neurosurgeon & Kolkata/NE \\
\hline Dr. Subhayon Mandal & Neurosurgeon & Kolkata/NE \\
\hline Dr. P K Sachdeva & Neurosurgeon & Delhi \\
\hline Dr. Minhaj Momin & Neurosurgeon & Kolkata/NE \\
\hline Dr. Manas Panigrahi & Neurosurgeon & Hyderabad \\
\hline Dr. B Rohit & Neurosurgeon & $\mathrm{TN}$ \\
\hline Dr. Rakesh Redhu & Neurosurgeon & Delhi \\
\hline Dr. R C Mishra & Neurosurgeon & Raj/UP \\
\hline
\end{tabular}




\begin{tabular}{|c|l|c|}
\hline Dr Chakaravarthi Avulla & Neurosurgeon & TN \\
\hline Dr Debabrata Biswal & Neurosurgeon & Kolkata/NE \\
\hline Dr Harish Ojha & Neurosurgeon & Kolkata/NE \\
\hline
\end{tabular}

\section{Acknowledgement}

All named authors meet the International Committee of Medical Journal Editors (ICMJE) criteria for authorship for this manuscript, take responsibility for the integrity of the work, and have given final approval for the version to be published. Medical writing and editorial support in the preparation of this article was provided by Dr. Punit Srivastava and Dr. Tarveen Jandoo of Mediception Science Pvt Ltd (www.mediception.com)

\section{Conflict of Interest}

None.

\section{References}

1. Yerram S, Katyal N, Premkumar K, Nattanmai P, Newey CR (2018) Seizure prophylaxis in the neuroscience intensive care unit. J Intensive Care 6: 17.

2. Temkin NR (2002) Prophylactic anticonvulsants after neurosurgery. Epilepsy Curr 2(4): 105-107.

3. North JB, Penhall RK, Hanieh A, Frewin DB, Taylor WB (1983) Phenytoin and postoperative epilepsy. A double blind study. J Neurosurg 58(5): 672-677.

4. Rapport RL, Penry JK (1973) A survey of attitudes toward the pharmacological prophylaxis of posttraumatic epilepsy. J Neurosurg; 38(2): 159-166.

5. Dauch WA, Schutze M, Guttinger M, Bauer BL (1996) Post-traumatic seizure prevention-results of a survey of 127 neurosurgery clinics. Zentralbl Neurochir 57(4): 190-195.

6. GreenhalghJ,Weston J,DundarY,NevittSJ, Marson AG (2013) Antiepileptic drugs as prophylaxis for post-craniotomy seizures. Cochrane Database Syst Rev 2018(5): CD007286.

7. US Food and Drug Administration (2016) Brivaracetam.

8. Gillard M, Fuks B, Leclercq K, Matagne A (2011) Binding characteristics of brivaracetam, a selective, high affinity SV2A ligand in rat, mouse and human brain: relationship to anti-convulsant properties. Eur J Pharmacol 664(1-3): 36-44.

9. (2020) U.S. Preventive services task force ratings.

10. Butzkueven H, Evans AH, Pitman A, Leopold C, Jolley DJ, et al. (2000) Onset seizures independently predict poor outcome after subarachnoid hemorrhage. Neurology 55(9): 1315-1320.

11. Habibabadi JM, Saadatnia M, Tabrizi N (2018) Seizure in cerebral venous and sinus thrombosis. Epilepsia Open 3(3): 316-322.

12. Doria JW, Forgacs PB (2019) Incidence, implications, and management of seizures following ischemic and hemorrhagic stroke. Curr Neurol Neurosci Rep 19(7): 37.

13. Englot DJ, Chang EF, Vecht CJ (2016) Epilepsy and brain tumors. Handb Clin Neurol 134: 267-285.

14. Connolly ES Jr, Rabinstein AA, Carhuapoma JR, Derdeyn CP, Dion J, et al. (2012) Guidelines for the management of aneurysmal subarachnoid hemorrhage: a guideline for healthcare professionals from the American Heart Association/american Stroke Association. Stroke 43(6): 17111737.

15. Zimmermann LL, Diaz-Arrastia R, Vespa PM (2016) Seizures and the role of anticonvulsants after traumatic brain injury. Neurosurg Clin $\mathrm{N}$ Am 27(4): 499-508.

16. Carney N, Totten AM, O’Reilly C, Ullman JS, Hawryluk GW, et al. (2017) Guidelines for the Management of Severe Traumatic Brain Injury, Fourth Edition. Neurosurgery 80(1): 6-15.

17. Soffietti R, Baumert BG, Bello L, von Deimling A, Duffau H, et al. (2010) European federation of neurological societies. Guidelines on management of low-grade gliomas: report of an EFNS-EANO task force. Eur J Neurol: 17(9): 1124-1133.

18. Kerkhof M, Dielemans JC, van Breemen MS, Zwinkels H, Walchenbach R, et al. (2013) Effect of valproic acid on seizure control and on survival in patients with glioblastoma multiforme. Neuro Oncol 15(7): 961-967.

19. Saria MG, Corle C, Hu J, Rudnick JD, Phuphanich S, et al. (2013) Retrospective analysis of the tolerability and activity of lacosamide in patients with brain tumors: clinical article. J Neurosurg 118(6):11831187.

20. Franceschetti S, Binelli S, Casazza M, Lodrini S, Panzica F, et al. (1990) Influence of surgery and antiepileptic drugs on seizures symptomatic of cerebral tumours. Acta Neurochir (Wien): 103(1-2): 47-51.

21. Iuchi T, Kuwabara K, Matsumoto M, Kawasaki K, Hasegawa $\mathrm{Y}$, et al. (2015) Levetiracetam versus phenytoin for seizure prophylaxis during and early after craniotomy for brain tumours: a phase II prospective, randomised study. J Neurol Neurosurg Psychiatry 86(10): 1158-1162.

22. Greenhalgh J, Weston J, Dundar Y, Nevitt SJ, Marson AG (2018) Antiepileptic drugs as prophylaxis for postcraniotomy seizures. Cochrane Database Syst Rev 5(5): CD007286.

23. Siomin V, Angelov L, Li L, Vogelbaum MA (2005) Results of a survey of neurosurgical practice patterns regarding the prophylactic use of antiepilepsy drugs in patients with brain tumors. J Neurooncol: 74(2): 211215.

24. Lwu S, Hamilton MG, Forsyth PA, Cairncross JG, Parney IF (2010) Use of peri-operative anti-epileptic drugs in patients with newly diagnosed high grade malignant glioma: a single center experience. J Neurooncol 96(3): 403-408.

25. Rizzo A, Donzelli S, Girgenti V, Sacconi A, Vasco C, et al. (2017) In vitro antineoplastic effects of brivaracetam and lacosamide on human glioma cells. J Exp Clin Cancer Res 36(1): 76.

26. Kruer RM, Harris LH, Goodwin H, Kornbluth J, Thomas KP, et al. (2013) Changing trends in the use of seizure prophylaxis after traumatic brain injury: a shift from phenytoin to levetiracetam. J Crit Care 28(5): 883. e9-883.e13.

27. Pourzitaki C, Tsaousi G, Apostolidou E, Karakoulas K, Kouvelas D, et al. (2016) Efficacy and safety of prophylactic levetiracetam in supratentorial brain tumour surgery: a systematic review and meta-analysis. Br J Clin Pharmacol 82(1): 315-325.

28. Szaflarski JP, Sangha KS, Lindsell CJ, Shutter LA (2010) Prospective, randomized, single-blinded comparative trial of intravenous levetiracetam versus phenytoin for seizure prophylaxis. Neurocrit Care 12(2): 165-712. 
29. Jones KE, Puccio AM, Harshman KJ, Falcione B, Benedict N, et al. (2008) Levetiracetam versus phenytoin for seizure prophylaxis in severe traumatic brain injury. Neurosurg Focus 25(4): E3.

30. Zhao L, Wu YP, Qi JL, Liu YQ, Zhang K, et al. (2018) Efficacy of levetiracetam compared with phenytoin in prevention of seizures in brain injured patients: A meta-analysis. Medicine (Baltimore) 97(48): e13247.

31. Rigo JM, Nguyen L, Hans G, Belachew S, Moonen G, et al. (2004) UCB 34714: effect on inhibitory and excitatory neurotransmission. Epilepsia: 45(s3): 56.

32. Niespodziany I, Lukyanetz EA, Matagne A, Klitgaard H, Wolff C (2017) Brivaracetam does not modulate ionotropic channels activated by glutamate, $\mathrm{\gamma}$-aminobutyric acid, and glycine in hippocampal neurons. Epilepsia: 58(11): e157-e161.

33. Kostyuk PG, Lukyanetz EA, Klitgaard H, Margineanu DG (2004) UCB 34714 , a new pyrrolidone derivative without impact on high and low voltage activated calcium currents in rat isolated neurons. Epilepsia: 45(7): 141

34. Rolan P, Sargentini-Maier ML, Pigeolet E, Stockis A (2008) The pharmacokinetics, CNS pharmacodynamics and adverse event profile of brivaracetam after multiple increasing oral doses in healthy men. Br J Clin Pharmacol 66(1): 71-75.

35. Klein P, Diaz A, Gasalla T, Whitesides J (2018) A review of the pharmacology and clinical efficacy of brivaracetam. Clin Pharmacol 10 $1-22$.

ISSN: 2574-1241

DOI: $10.26717 /$ BJSTR.2021.38.006154

Kumar Gaurav. Biomed J Sci \& Tech Res

(C) (P) This work is licensed under Creative Commons Attribution 4.0 License

Submission Link: https://biomedres.us/submit-manuscript.php
36. Finnema SJ, Mercier J, Naganawa M, Henry S, Gao H, et al. (2017) Brivaracetam enters the brain faster than levetiracetam: a PET study in healthy volunteers. Neurology 88(16): P6.233.

37. Klein P, Johnson ME, Schiemann J, Whitesides J (2017) Time to onset of sustained $\geq 50 \%$ responder status in patients with focal (partial-onset) seizures in three phase III studies of adjunctive brivaracetam treatment. Epilepsia 58(2): e21-e25.

38. Witt JA, Elger CE, Helmstaedter C (2018) Short-term and longer-term effects of brivaracetam on cognition and behavior in a naturalistic clinical setting-Preliminary data. Seizure 62: 49-54.

39. Chen Z, Lusicic A, O’Brien TJ, Velakoulis D, Adams SJ, et al. (2016) Psychotic disorders induced by antiepileptic drugs in people with epilepsy. Brain 139(10): 2668-2678.

40. Weintraub D, Buchsbaum R, Resor SR Jr, Hirsch LJ (2007) Psychiatric and behavioral side effects of the newer antiepileptic drugs in adults with epilepsy. Epilepsy Behav 10(1): 105-110.

41. Sarkis RA, Goksen Y, Mu Y, Rosner B, Lee JW (2018) Cognitive and fatigue side effects of anti-epileptic drugs: an analysis of phase III add-on trials. J Neurol 265(9): 2137-2142.

$\begin{array}{ll}\text { BIOMEDICAL } & \text { Assets of Publishing with us } \\ \text { RESEARCHES } & \text { - Global archiving of articles } \\ & \text { - Immediate, unrestricted online access } \\ & \text { - Rigorous Peer Review Process } \\ & \end{array}$

